\title{
Renal and Cardiovascular
} Morbidities Associated with APOL1 Status among African-American and Non-African-American Children with Focal Segmental Glomerulosclerosis

OPEN ACCESS

Edited by:

Max Christoph Liebau, University Hospital of Cologne,

Germany

Reviewed by:

Walter Gabriel Wasser,

Albert Einstein College of Medicine,

USA

Miriam Schmidts,

Radboud University Nijmegen,

Netherlands

${ }^{*}$ Correspondence:

Robert P. Woronieck

robert.woroniecki@

stonybrookmedicine.edu

Specialty section:

This article was submitted to

Pediatric Nephrology,

a section of the journal

Frontiers in Pediatrics

Received: 04 August 2016

Accepted: 28 October 2016

Published: 17 November 2016

Citation:

Woroniecki RP, Ng DK, Limou S, Winkler CA, Reidy KJ, Mitsnefes M,

Sampson MG, Wong CS,

Warady BA, Furth SL, Kopp JB and

Kaskel FJ (2016) Renal and

Cardiovascular Morbidities

Associated with APOL1 Status

among African-American and Non-African-American Children with Focal Segmental Glomerulosclerosis.

Front. Pediatr. 4:122.

doi: 10.3389/fped.2016.00122

\author{
Robert P. Woroniecki ${ }^{*}$, Derek K. Ng ${ }^{2}$, Sophie Limou ${ }^{3}$, Cheryl A. Winkler ${ }^{3}$, \\ Kimberly J. Reidy ${ }^{4}$, Mark Mitsnefes ${ }^{5}$, Matthew G. Sampson ${ }^{6}$, Craig S. Wong, \\ Bradley A. Warady ${ }^{8}$, Susan L. Furth ${ }^{9}$, Jeffrey B. Kopp ${ }^{10}$ and Frederick J. Kaskel ${ }^{4}$
}

'Stony Brook Children's Hospital, Stony Brook, NY, USA, ${ }^{2}$ Johns Hopkins Bloomberg School of Public Health, Baltimore, MD, USA, ${ }^{3}$ Basic Research Laboratory, Frederick National Laboratory, NCI, NIH, Leidos Biomedical, Frederick, MD, USA ${ }^{4}$ Pediatric Nephrology, Children's Hospital at Montefiore, Bronx, NY, USA, ${ }^{5}$ Division of Nephrology and Hypertension, Cincinnati Children's Hospital Medical Center, Cincinnati, OH, USA, ${ }^{6}$ Division of Pediatric Nephrology, University of Michigan School of Medicine, Ann Arbor, MI, USA, 'Pediatric Nephrology, University of New Mexico, Albuquerque, NM, USA, ${ }^{8}$ Division of Pediatric Nephrology, Children's Mercy Hospital, Kansas City, MO, USA, ${ }^{9}$ University of Pennsylvania, Philadelphia, PA, USA, ${ }^{10} \mathrm{NIDDK}, \mathrm{NIH}$, Bethesda, MD, USA

Background and objectives: African-American (AA) children with focal segmental glomerulosclerosis (FSGS) have later onset disease that progresses more rapidly than in non-AA children. It is unclear how APOL 1 genotypes contribute to kidney disease risk, progression, and cardiovascular morbidity in children.

Design, setting, participants, and measurements: We examined the prevalence of APOL1 genotypes and associated cardiovascular phenotypes among children with FSGS in the Chronic Kidney Disease in Children (CKiD) study; an ongoing multicenter prospective cohort study of children aged 1-16 years with mild to moderate kidney disease.

Results: A total of 140 AA children in the CKiD study were genotyped. High risk (HR) APOL 1 genotypes were present in $24 \%$ of AA children (33/140) and were associated with FSGS, $p<0.001$. FSGS was the most common cause of glomerular disease in children with HR APOL1 (89\%; 25/28). Of 32 AA children with FSGS, 25 (78\%) had HR APOL 1. Compared to children with low risk APOL1 and FSGS (comprising 36 non-AA and 7 AA), children with HR APOL1 developed FSGS at a later age, 12.0 (IQR: 9.5, $12.5)$ vs. $5.5(2.5,11.5)$ years, $p=0.004$, had a higher prevalence of uncontrolled hypertension (52 vs. 33\%, $p=0.13$ ), left ventricular hypertrophy (LVH) (53 vs. $12 \%$, $p<0.01$ ), C-reactive protein $>3 \mathrm{mg} / \mathrm{l}$ (33 vs. $15 \%, p=0.12$ ), and obesity (48 vs. $19 \%$, $p=0.01$ ). There were no differences in glomerular filtration rate, hemoglobin, $\mathrm{PTH}$, or calcium-phosphate product.

Conclusion: AA children with HR APOL 1 genotype and FSGS have increase prevalence of obesity and LVH despite a later age of FSGS onset, while adjusting for socioeconomic status. Treatment of obesity may be an important component of chronic kidney disease and LVH management in this population.

Keywords: cardiovascular, left ventricular hypertrophy, chronic renal disease, FSGS, children 


\section{INTRODUCTION}

African-Americans (AA) have higher rates of hypertension (HTN) and kidney disease compared to Americans of European descent (1). In adults of African descent, the presence of high risk (HR) APOL1 genotype (characterized by the presence of two risk alleles, defined as G1/G1 homozygotes, G2/G2 homozygotes, and G1/G2 compound heterozygotes), preferentially selected by the process of evolution, was found to be associated with nondiabetic or "hypertension-attributed" end-stage renal disease (ESRD), idiopathic focal segmental glomerulosclerosis (FSGS), and HIV-associated nephropathy (2-5). The role of APOL1 in the adult cardiovascular phenotype is still controversial with some recent findings, suggesting that APOL1 variants could contribute to atherosclerotic cardiovascular risk, indicating a genetic component to cardiovascular health disparities among adults of African ancestry (6). While studies in adult AA populations demonstrated strong recessive association of APOL1 G1 and G2 genetic variants with glomerular and vascular disease progression (7), there is limited information on its role in children with chronic kidney disease (CKD), particularly for cardiovascular comorbidities. In NIH FSGS cohort study and in the FSGSClinical Trial (FSGS-CT), both of which included children and adults, HR APOL1 genotype was present in $72 \%$ of self-identified AA subjects (8).

Up to $63 \%$ of children with early stages of CKD present with arterial HTN (9). In addition to being common, HTN is associated with a greater rate of decline in kidney function and is a known risk of development of ESRD (10). Furthermore, in Chronic Kidney Disease in Children (CKiD), a population with mild to moderate $\mathrm{CKD}$, a high overall prevalence (53\%) of systolic HTN was observed (11). Presence of HTN in children with CKD has also been associated with cardiovascular morbidity and development of left ventricular hypertrophy (LVH) (12). LVH is common among hypertensive adults and children with CKD and is more common among AA than in whites $(13,14)$. While a previous report of two pediatric cohorts representing children with CKD (CKiD) and nephrotic syndrome (Nephrotic Syndrome Study Network; NEPTUNE) showed similar APOL1 characteristics in terms of FSGS diagnosis and disease progression (15), the association of APOL1 and cardiovascular comorbidities in the presence of CKD has not been explored. The purpose of this study was to extend previous findings (15), to characterize the distribution of APOL1 risk alleles in the CKiD cohort and, by comparing children with an underlying FSGS cause of CKD, to describe the prevalence of cardiorenal phenotypes and markers of disease severity associated with the HR APOL1 genotype.

\section{MATERIALS AND METHODS}

\section{Subjects}

The study population comprised of children enrolled in the CKiD Study, an ongoing multicenter prospective cohort study of children aged 1-16 years with mild to moderate kidney disease (16).

\section{Clinical and Laboratory Testing}

All clinical and laboratory data in the analysis were based on the first available observation, either at study entry or 6 months after, and were categorized by indicators of renal, cardiovascular, and metabolic health. Echocardiography data were collected 1 year after study entry according to study protocol. Race, sociodemographic, and therapy use were self or parental reported.

\section{Genetic Testing}

DNA was obtained from National Institute of Diabetes and Digestive and Kidney Disease (NIDDK) genetics repository of immortalized lymphocyte cell lines based on a sample of whole blood taken at the 6-month-old study visit among those who consented to genetic testing. APOL1 variants G1 (rs73885319, S342G and rs60945101, I384M) and G2 (rs71785313, NY deletion) were genotyped.

The number of kidney risk alleles defined APOL1 risk groups: low risk (LR) was defined by 0 or 1 risk allele (G0/G0 homozygous, or G1/G0 and G2/G0 heterozygous), and HR was defined by 2 risk alleles (G1/G1 and G2/G2 homozygous, or G1/ G2 compound heterozygous), where G0 represents the ancestral alleles at both rs73885319 and rs71785313 sites.

Indicators of renal health included glomerular filtration rate (GFR) measured by plasma disappearance of iohexol or estimated by CKiD-developed equations based on serum creatinine, cystatin C, and BUN, when iohexol GFR was not available. Proteinuria was assessed as first morning urine protein (milligrams)/creatinine (milligrams) ratio (uPCR).

Indicators of cardiovascular health included the average of three in-clinic measurements of blood pressure and left ventricular mass index (LVMI in $\mathrm{g} / \mathrm{m}^{2.7}$ ) as measured by echocardiography. Uncontrolled HTN was defined as systolic blood pressure (SBP) or diastolic blood pressure (DBP) $\geq 95$ th percentile for age, sex, height according to the fourth report (17). LVH was defined as LVMI $\geq$ age- and sex-specific 95 th percentile based on the normal population (18). All data were measured and defined by the CKiD study protocol, as described previously $(11,16,19)$.

Indicators of metabolic health included total, HDL, and LDL cholesterol (milligrams per deciliter), triglycerides (milligrams per deciliter) with age- and sex-defined categorical definitions for abnormally high total cholesterol, low HDL, and high LDL cholesterol, based on the normal population. Hemoglobin (grams per deciliter), calcium (milligrams per deciliter), phosphate (milligrams per deciliter), and calcium $\times$ phosphate product were measured. Hemoglobin was categorized by anemia status based on age- and sex-defined levels of the normal population, and phosphate levels were categorized as high based on age-defined levels of the normal population. Intact parathyroid hormone (iPTH) and C-reactive protein (CRP) were also measured (19), with high sensitivity CRP primarily used $(n=47)$ and when missing, informed by wide-range CRP data $(n=18$; missing both $n=3)$. High CRP was defined as CRP $>3 \mathrm{mg} / \mathrm{l}$.

The CKiD Study protocol was approved by the institutional review boards at the participating institutions, and all subjects gave informed assent or consent. Genetic testing was approved by the NIDDK Institutional Review Board. 


\section{Statistical Analyses}

Since $89 \%$ of children with the HR APOL1 profile had FSGS, the appropriate comparison group to determine differences related to APOL1 were children with LR APOL1 genotype and FSGS. Fisher's exact test for categorical variables and the Wilcoxon rank-sum test for continuous variables were used to compare univariate differences by $A P O L 1$ risk status among those with a diagnosis of FSGS.

Since lower socioeconomic status (SES) was associated with AA race in this North American cohort, and low SES is associated with disease progression (20), SES is a potential confounder as the exposure groups largely differ by race. To adjust for confounding due to SES, we used inverse probability of exposure weights (IPWs) $(21,22)$. The IPWs were generated from a logistic regression model with HR APOL1 as the outcome and variables related to SES as the predictors: income less than $\$ 36,000$, maternal education less than college, the presence of any public insurance and missing at least one dose of antihypertensive therapy in the past 7 days (self-reported). The inverse of the predicted probability of observed exposure was stabilized to the marginal probability of each group (for HR APOL1: 25/68 = 0.37; and for LR APOL1: $43 / 68=0.63)$. Selected cardiovascular, metabolic, inflammatory, and other risk factors were compared to exposure group in adjusted analyses using weighting by IPWs in logistic regression models to obtain prevalence odds ratio comparing the HR APOL1 group to the LR APOL1 group. The outcomes were obesity, high LDL cholesterol, uncontrolled HTN, LVH, and CRP > $3 \mathrm{mg} / \mathrm{l}$. Multiple imputation was used to account for missing data, and the results are presented unadjusted (univariately, with imputation where applicable) and adjusted to account for the effect of confounding by SES.

\section{RESULTS}

A total of 891 children were enrolled in CKiD, of whom 199 (22\%) were AA; Figure 1 presents the distributions of APOL1 genotypes and CKD diagnoses by race. Among 140 genotyped AA children, HR APOL1 status was present in 33 (24\%), and among these 33 individuals 28 (85\%) had an underlying glomerular cause of CKD, whereas only $5 / 33(6 \%)$ had a non-glomerular cause of CKD, $p<0.001$. Of those 28 subjects with HR APOL1 status and a glomerular disease, $25 / 28$ (89\%) had a diagnosis of FSGS. In contrast, among 191 non-AA children with a glomerular CKD cause, only $36(19 \%)$ had an FSGS diagnosis, $p<0.001$. Of the 28 AA children with LR APOL1 status (defined as presence of 0 or 1 risk allele), and glomerular CKD, 7 (25\%) had a diagnosis of FSGS (difference between HR and LR APOL1 AA, $p<0.001$ ). $A P O L 1$ genotyping was not performed in 59 children: 20 did not consent to genetic testing, 19 dropped out of the study prior to sample collections, 9 were not measured due to missing samples, and 11 samples became available only after genotyping had been completed. Of these 59 children, $47 \%$ had a glomerular cause of CKD; $39 \%$ of these had a diagnosis of FSGS. The similar proportions of glomerular diagnoses and FSGS among AA LR APOL1 and non-AA LR APOL1 children (i.e., 25 and 19\%, respectively) suggest potentially similar pathologic mechanisms and were distinct from the distributions observed among AA children with HR APOL1. The proportion (47\%) of FSGS diagnoses among children with unmeasured APOL1 status is intermediate between that observed among those with HR (85\%) and LR (25\%), suggesting that this group with unmeasured genotypes comprises both HR and LR profiles. A total of 37 non-AA children were genotyped, and all were identified as having LR APOL1 status

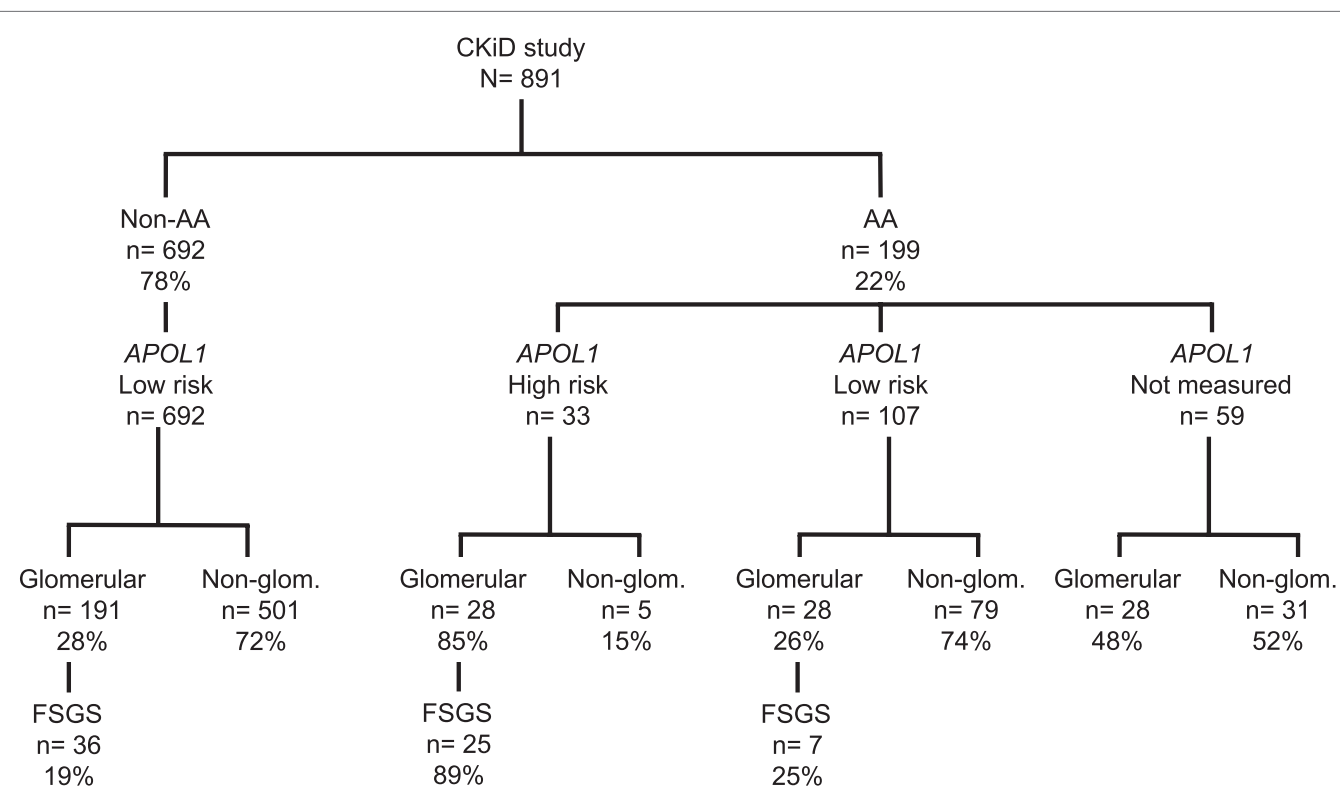

FIGURE 1 | Distribution of CKiD children by race [African-American (AA) vs. non-African-American (non-AA)], APOL1 genotypes, and CKD diagnoses [glomerular disease (Glom), non-glomerular disease (Non-Glom), focal segmental glomerulosclerosis (FSGS)] All genotyped non-AA ( $N=37$ ) were found to have LR APOL1 status, providing a justification for classifying non-AA children as low risk. 
(36 had 0 risk alleles and 1 harbored 1 risk allele). This provided justification for classifying non-AA children as LR. A full list of diagnoses by race and APOL1 status is provided in Table S1 in Supplementary Material.

We compared the prevalence of renal and cardiovascular risk factors by APOL1 status and AA race within FSGS diagnosis group $(n=68)$. Since there were only seven subjects with LR APOL1 and AA race, we pooled both AA $(n=7)$ and non-AA $(n=36)$ children as the reference population for statistical comparison with AA children with HR APOL 1 and FSGS $(n=25)$. Table 1 presents the clinical, sociodemographic, and renal characteristics among those with FSGS, stratified by race and APOL1 risk status. There were similar proportions of boys and despite similar ages at study entry, those with HR APOL1 were significantly taller $(p=0.003)$ and heavier $(p<0.001)$, compared to children with LR APOL1.
This was reflected in the height and weight percentiles standardized to age and gender (i.e., normal, median height, and weight percentiles are equal to 50): those with HR APOL1 had a median height and weight percentiles significantly higher as compared to those with LR APOL1, $p=0.033$ and $p<0.001$, respectively. Body mass index (BMI) was significantly higher among the HR APOL1 children $(p<0.001)$, and this group had a much higher proportion of obesity $(\mathrm{BMI}>85$ th percentile adjusted for age and gender; 48 vs. $19 \%, p=0.01$ ). Consistent with previously published reports (15), children with HR APOL1 had a higher prevalence of premature birth than children with LR APOL1 (29 vs. $5 \%, p=0.011$ ), but this difference was not observed for low birth weight or small for gestational age.

To account for the potential confounding effects of SES factors related to race, inverse probability weights were used to adjust

TABLE 1 | Descriptive statistics of sociodemographic, renal health, and therapy use among children with FSGS, by race and APOL1 risk status (LR, low risk vs. HR, high risk; non-AA, non-African-American; AA, African-American), in the CKiD study ( $n=68$ ), median (IQR), $n$ (\%); $p$-values based on Fisher's exact test or Wilcoxon rank-sum test.

\begin{tabular}{|c|c|c|c|c|c|}
\hline Variable & $\begin{array}{c}\text { Non-AA APOL1 LR } \\
n=36\end{array}$ & $\begin{array}{c}\text { AA APOL1 LR } \\
n=7\end{array}$ & $\begin{array}{l}\text { Pooled APOL1 LR } \\
\qquad n=43\end{array}$ & $\begin{array}{c}A P O L 1 \mathrm{HR} \\
n=25\end{array}$ & $\begin{array}{c}p \text {-Value } \\
\text { (pooled LR vs. HR) }\end{array}$ \\
\hline Male & $21(58 \%)$ & $4(57 \%)$ & $25(58 \%)$ & $11(44 \%)$ & 0.318 \\
\hline Black race & 0 & 7 (100\%) & 7 (16\%) & $25(100 \%)$ & NA \\
\hline Age at study entry, years & $13.8[9.6,15.6]$ & $15.0[8.2,16.5]$ & $14.2[9.5,15.8]$ & $14.8[13.0,15.5]$ & 0.312 \\
\hline Low birth weight $(<2500 \mathrm{~g})$ & $7(21 \%)$ & $1(17 \%)$ & $8(21 \%)$ & $8(33 \%)$ & 0.372 \\
\hline Premature & $2(6 \%)$ & $0(0 \%)$ & $2(5 \%)$ & $7(29 \%)$ & 0.011 \\
\hline Small for gestational age & $7(23 \%)$ & $2(33 \%)$ & $9(24 \%)$ & 7 (30\%) & 0.765 \\
\hline $\begin{array}{l}\text { Low birth weight, premature, or } \\
\text { small for gestational age }\end{array}$ & $10(29 \%)$ & $2(29 \%)$ & $12(29 \%)$ & $13(52 \%)$ & 0.070 \\
\hline Height, cm & $147[135,163]$ & $147[128,160]$ & $147[135,163]$ & $164[149,171]$ & 0.003 \\
\hline Height percentile & $24[4,59]$ & $14[1,47]$ & $23[3,55]$ & $40[27,86]$ & 0.033 \\
\hline Weight, kg & $46.4[34.4,56.5]$ & $47.1[27.4,72.4]$ & $46.4[34.0,57.3]$ & $73.5[60.4,90.6]$ & $<0.001$ \\
\hline Weight percentile & $53[20,77]$ & $59[14,91]$ & $54[18,77]$ & $97[71,99]$ & $<0.001$ \\
\hline Body mass index, $\mathrm{kg} / \mathrm{m}^{2}$ & $20.2[17,23.9]$ & $19.4[16.6,27.1]$ & $20[17.4,24.1]$ & $25.1[22.9,36.3]$ & $<0.001$ \\
\hline BMI percentile & $74[28,93]$ & $77[38,91]$ & $76[33,93]$ & $93[82,99]$ & $<0.001$ \\
\hline Obese & 7 (19\%) & $1(14 \%)$ & $8(19 \%)$ & $12(48 \%)$ & 0.014 \\
\hline \multicolumn{6}{|l|}{ Socioeconomic variables } \\
\hline \multicolumn{6}{|l|}{ Household income } \\
\hline$<\$ 36,000$ & $15(43 \%)$ & $2(33 \%)$ & $17(41 \%)$ & $12(48 \%)$ & 0.409 \\
\hline$\geq \$ 36,000$ and $<\$ 75,000$ & $11(31 \%)$ & $2(33 \%)$ & $13(32 \%)$ & $10(40 \%)$ & \\
\hline$\geq \$ 75,000$ & $9(26 \%)$ & $2(33 \%)$ & $11(27 \%)$ & $3(12 \%)$ & \\
\hline Maternal education < college & $26(74 \%)$ & $6(86 \%)$ & 32 (76\%) & $17(68 \%)$ & 0.571 \\
\hline Any public insurance & $19(53 \%)$ & $2(29 \%)$ & $21(49 \%)$ & $15(60 \%)$ & 0.453 \\
\hline \multicolumn{6}{|l|}{ Renal health } \\
\hline Age at CKD onset, years & $6.5[2.5,11.5]$ & $4.5[3.5,3.5]$ & $5.5[2.5,11.5]$ & $12.0[9.5,12.5]$ & 0.004 \\
\hline Years with CKD & $5.3[3.4,7.9]$ & $3.7[2.2,2.3]$ & $5.2[3.3,7.9]$ & $3.3[1.1,4.7]$ & 0.008 \\
\hline ieGFR at entry, ml/min/1.73 m² & $48[34,71]$ & $32[26,98]$ & $48[32,79]$ & $61[48,69]$ & 0.132 \\
\hline leGFR $<45 \mathrm{ml} / \mathrm{min} / 1.73 \mathrm{~m}^{2}$ & $14(39 \%)$ & $4(57 \%)$ & $18(42 \%)$ & $6(24 \%)$ & 0.190 \\
\hline ieGFR change per year, \% & $-7.4 \%[-3.1 \%,-2.9 \%]$ & $-2.2 \%[-14.0 \%,-14.2 \%]$ & $-7.4 \%[-23.0 \%,-2.9 \%]$ & $-8.3 \%[-14.9 \%,-1.7 \%]$ & 0.994 \\
\hline ieGFR change per year, ml/min & $-4.1[-12.3,-1.7]$ & $-2.5[-7.8,5.9]$ & $-4.8[-12.8,-1.7]$ & $-4.4[-9.6,-1.1]$ & 0.903 \\
\hline uPCR at entry, mg/mg creatinine & $1.6[0.2,5.5]$ & $1.0[0.1,1.2]$ & $1.5[0.2,5.5]$ & $0.9[0.3,1.8]$ & 0.330 \\
\hline Proteinuria, $\mathrm{UPCR}>2$ & $15(43 \%)$ & $3(43 \%)$ & $18(43 \%)$ & $3(13 \%)$ & 0.025 \\
\hline \multicolumn{6}{|l|}{ Therapy use } \\
\hline Anti-hypertension therapy & $31(86 \%)$ & $7(100 \%)$ & 38 (88\%) & 25 (100\%) & 0.150 \\
\hline ACEi/ARB therapy & $29(81 \%)$ & 7 (100\%) & $36(84 \%)$ & $22(88 \%)$ & 0.735 \\
\hline Missed ACEi/ARB in last 30 days & $7(19 \%)$ & $1(14 \%)$ & $8(19 \%)$ & $4(16 \%)$ & 1.000 \\
\hline Missed ACEi/ARB in last 7 days & $6(17 \%)$ & $3(43 \%)$ & $9(21 \%)$ & $10(40 \%)$ & 0.103 \\
\hline Steroid therapy & 7 (19\%) & $2(29 \%)$ & $9(21 \%)$ & $8(32 \%)$ & 0.387 \\
\hline Immunosuppression therapy & $17(47 \%)$ & $5(71 \%)$ & $22(51 \%)$ & $14(56 \%)$ & 0.803 \\
\hline
\end{tabular}

$B M I$, body mass index; CKD, chronic kidney disease; ieGFR, measured or estimated glomerular filtration rate; uPCR, urine protein to creatinine ratio. 


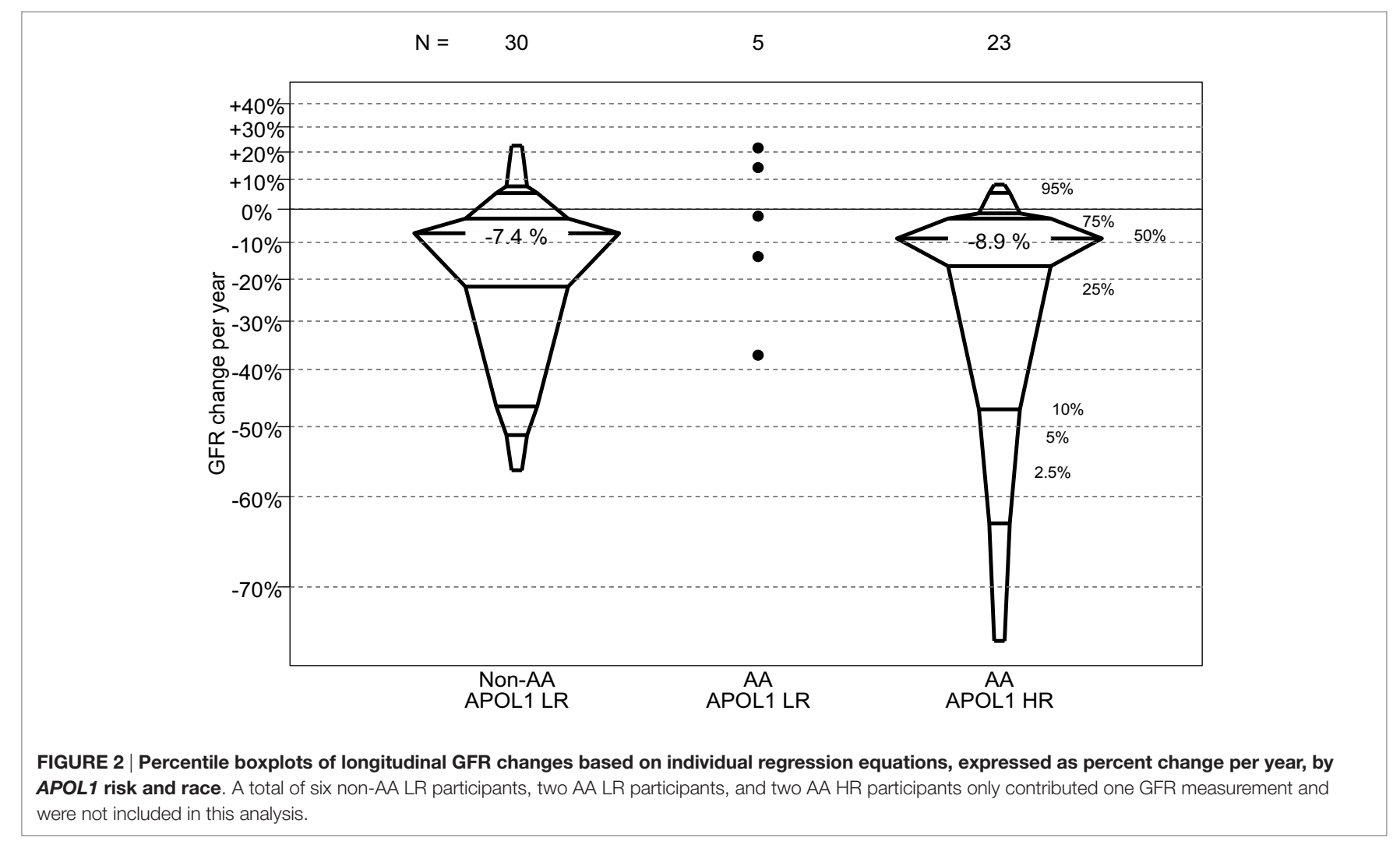

for sex, income, maternal education, insurance status, and 7-day adherence to antihypertensive therapy. We found no statistical differences in SES variables between HR and LR APOL1 genotype groups.

In this comparison, those children with FSGS and HR APOL1 had a significantly older age at CKD onset, and significantly shorter duration of CKD than those with LR APOL1. Children with HR APOL1 had a higher GFR at entry although this difference was not statistically significant, $p=0.132$. Changes in GFR over time were similar to APOL1 status (Figure 2). Levels of uPCR at entry and over time were also similar, although the HR APOL1 group had a lower proportion with nephrotic range proteinuria at study entry, $p=0.025$.

Table 2 and Table S2A,B in Supplementary Material describe characteristics related to cardiovascular health stratified by race and APOL1 risk status among those with an FSGS cause of CKD. Children with HR APOL1 had higher SBP $(p=0.004)$, although SBP percentiles (standardized to the normal population for age, gender, and height) were not statistically significant $(p=0.132)$. No significant differences in DBP were observed. The HR APOL1 children had a higher prevalence of uncontrolled HTN, although it was not statistically significant. Nearly all children, regardless of APOL1 status received antihypertensive therapy, the HR APOL1 group had higher (40 vs. $21 \%$ ) 7-day non-adherence to ACEi/ ARB therapy, $p=0.103$ (Table 1). The proportions receiving glucocorticoid and other immunosuppression therapy were similar by $A P O L 1$ risk status.

Increased left ventricular mass index $(p=0.004)$ and a higher prevalence of LVH $(p=0.003)$ were observed in the HR APOL1 group. LDL cholesterol was higher in the HR APOL1 group $(p=0.047)$, but no differences in total cholesterol, HDL cholesterol, and triglyceride levels were observed. Higher phosphate was observed among the LR APOL1 group $(p=0.023)$, but there was no difference in intact parathyroid hormone. Children with HR APOL1 had a higher proportion of CRP values greater than $3 \mathrm{mg} / \mathrm{l}$ (33 vs. $15 \%, p=0.120$ ).

Table 3 presents odds ratios to describe the associations of the HR APOL1 profile and selected risk factors and comorbidities. LVH was highly associated with HR APOL1 (OR: 6.2, 95\%CI: 1.6, 24.9), as was obesity (OR: 4.7, 95\%CI: 1.5, 14.4). While not statistically significant, HR APOL 1 had a higher relative odds of uncontrolled HTN, elevated CRP, and high LDL cholesterol.

\section{DISCUSSION}

In this cohort of children with FSGS, we demonstrated that AA children with HR APOL1 genotype were more likely to have FSGS and to have a later age at FSGS onset, compared to AA children with LR genotypes. When restricting analysis to children with FSGS, HR APOL1 was associated with a greater cardiovascular risk burden, including an increase prevalence of $\mathrm{LVH}$, and obesity, a higher LVMI, BMI, and LDL cholesterol, despite treatment with antihypertensive therapy and adjustment for indicators of SES. Notably, other markers of disease severity were not different between the two groups indicating a potential role for APOL1 in the etiology of cardiovascular abnormalities. 
TABLE 2 | Cardiovascular and metabolic characteristics among children with FSGS, by race and APOL1 status.

\begin{tabular}{|c|c|c|c|c|c|}
\hline Variable & $\begin{array}{c}\text { Non-AA APOL1 LR } \\
n=36\end{array}$ & $\begin{array}{c}\text { AA APOL1 LR } \\
n=7\end{array}$ & $\begin{array}{c}\text { Pooled APOL1 LR } \\
n=43\end{array}$ & $\begin{array}{c}\text { APOL1 HR } \\
n=25\end{array}$ & $\begin{array}{c}p \text {-Value } \\
\text { (pooled LR vs. HR) }\end{array}$ \\
\hline SBP, mmHg & $108[103,117]$ & $114[109,124]$ & $109[103,119]$ & $120[113,127]$ & 0.004 \\
\hline SBP percentile & $62[43,81]$ & $88[48,97]$ & $64[45,88]$ & $79[55,95]$ & 0.132 \\
\hline DBP, mmHg & $66[61,77]$ & $73[67,88]$ & $69[61,79]$ & $67[63,75]$ & 0.889 \\
\hline DBP percentile & $64[36,88]$ & $90[58,99]$ & $68[37,92]$ & $57[46,85]$ & 0.377 \\
\hline Uncontrolled hypertension & $10(28 \%)$ & $4(57 \%)$ & $14(33 \%)$ & $13(52 \%)$ & 0.131 \\
\hline LVMI at V2, $\mathrm{g} / \mathrm{m}^{2.7}$ & $30.0[26.9,33.0]$ & $28.1[20.7,28.6]$ & $29.6[26.9,33.0]$ & $40.8[28.1,52.9]$ & 0.004 \\
\hline LVH at V2 & $4(13.8 \%)$ & $0(0 \%)$ & $4(12 \%)$ & $9(45 \%)$ & 0.003 \\
\hline Total cholesterol, mg/dl & $173[150,210]$ & $186[174,205]$ & $174[150,210]$ & $190[168,224]$ & 0.222 \\
\hline High total cholesterol & $10(33 \%)$ & $2(40 \%)$ & $12(34 \%)$ & $9(43 \%)$ & 0.565 \\
\hline HDL cholesterol, mg/dl & $53[41,61]$ & $53[50,66]$ & $53[41,63]$ & $51[41,60]$ & 0.883 \\
\hline Low HDL cholesterol & $4(13 \%)$ & $0(0 \%)$ & $4(11 \%)$ & $0(0 \%)$ & 0.286 \\
\hline LDL cholesterol, mg/dl & $90[71,120]$ & $98[97,123]$ & $92[71,122]$ & $112[101,145]$ & 0.047 \\
\hline High LDL cholesterol & $5(17 \%)$ & $1(20 \%)$ & $6(17 \%)$ & 7 (33\%) & 0.208 \\
\hline Triglycerides, mg/dl & $143[92,203]$ & $118[93,160]$ & $136[92,200]$ & $117[91,145]$ & 0.441 \\
\hline High triglycerides & $18(60 \%)$ & $3(60 \%)$ & $21(60 \%)$ & $10(48 \%)$ & 0.578 \\
\hline Hemoglobin, g/dl & $12.5[11.3,13.4]$ & $11.7[10.0,12.9]$ & $12.3[11.1,13.3]$ & $12.4[11.9,13.6]$ & 0.165 \\
\hline Anemia & $17(47 \%)$ & $5(71 \%)$ & $22(51 \%)$ & $8(32 \%)$ & 0.139 \\
\hline Calcium, mg/dl & $9.2[8.5,9.6]$ & $9.6[8.0,9.8]$ & $9.2[8.4,9.6]$ & $9.4[9.1,9.7]$ & 0.179 \\
\hline Phosphate (mg/dl) & $4.5[4.0,5.1]$ & $4.1[3.7,6.0]$ & $4.4[4.0,5.1]$ & $4.3[3.7,4.7]$ & 0.154 \\
\hline High phosphate & $6(17 \%)$ & $2(29 \%)$ & $8(19 \%)$ & $0(0 \%)$ & 0.023 \\
\hline Calcium $\times$ phosphate & $41.7[37.4,44.8]$ & $41.0[36.3,48.0]$ & $41.4[37.0,45.0]$ & $39.9[34.0,45.6]$ & 0.504 \\
\hline iPTH, pg/ml & $58.0[35.8,89.8]$ & $144.3[40.0,267.8]$ & $58.0[38.0,95.0]$ & $52.0[39.5,67.0]$ & 0.399 \\
\hline $\mathrm{CRP}>3 \mathrm{mg} / \mathrm{l}$ & $4(12 \%)$ & $2(29 \%)$ & $6(15 \%)$ & $8(33 \%)$ & 0.120 \\
\hline
\end{tabular}

Median [IQR] or (CI), n (\%); p-values based on Fisher's exact test or Wilcoxon rank-sum test.

SBP, systolic blood pressure; DBP, diastolic blood pressure; LVMI, left ventricular mass index; LVH, left ventricular hypertrophy; V2, visit at 12 months post study enrollment; HDL, high density lipoprotein; LDL, low density lipoprotein; iPTH, intact parathyroid hormone; CRP, C-reactive protein (high sensitivity or wide range).

Uncontrolled hypertension was defined as SBP or DBP $\geq 95$ th percentile for age, sex, and height, regardless of a self-reported history of hypertension or receiving antihypertensive therapy.

TABLE 3 | Relative odds of selected risk factors and comorbidities comparing AA children with FSGS and HR APOL1 to pooled children (i.e. non-AA and AA) with FSGS and LR APOL1 based on logistic regression models.

\begin{tabular}{lcr}
\hline & $\begin{array}{c}\text { Unadjusted odds ratios } \\
\text { (95\% } \mathbf{C l})\end{array}$ & \multicolumn{1}{c}{$\begin{array}{c}\text { Adjusted odds ratios } \\
(\mathbf{9 5 \%} \mathbf{C l})\end{array}$} \\
\hline Left ventricular hypertrophy & $7.97(1.90,33.51)$ & $6.22(1.55,24.91)$ \\
Obesity & $4.04(1.35,12.11)$ & $4.65(1.50,14.43)$ \\
Uncontrolled hypertension & $2.24(0.82,6.17)$ & $2.54(0.92,7.00)$ \\
C-reactive protein > 3 mg/l & $2.74(0.82,9.16)$ & $2.41(0.67,8.72)$ \\
High LDL cholesterol & $1.34(0.44,4.08)$ & $1.22(0.40,3.72)$ \\
\hline
\end{tabular}

Analyses are unadjusted and adjusted for sex, income less than $\$ 30,000$, maternal education less than college, household having any public insurance, and missing a dose of antihypertensive medication in the past 7 days, as markers of socioeconomic status. Multiple imputation was used to account for missing data.

LDL, low density lipoprotein.

In children with biopsy confirmed FSGS enrolled in the FSGS-CT, $72 \%$ of self-identified AA subjects and $6 \%$ of children who identified themselves as non-AA had HR APOL1 risk alleles (8). In the CKiD population, HR APOL1 riskgenotype was strongly associated with both glomerular disease and FSGS. Strikingly, the prevalence of HR APOL1 among AA children with FSGS was 76\% (25/33), and this was congruent with the $72 \%$ observed in the FSGS-CT. The distribution of APOL1 genotypes observed in the adult normal population did not differ from Hardy-Weinberg expectations for genotype distribution; there was no loss of one particular genotype group or gain in another. This provides population-based statistical evidence that there is no preferential loss of HR genotypes between conception and adulthood (2-5). While our study focused on children who have already been diagnosed with CKD, we are unable to make inferences about the general (i.e., non-diseased) pediatric population. Future studies may wish to characterize the distribution of APOL1 risk alleles in this group to better understand population risk in children.

Previous studies have established that APOL1 kidney risk variants are strongly associated with kidney disease among adult AA; specifically, HIV-associated nephropathy (odds ratio, 29; 95\% confidence interval, 13-68) and FSGS (odds ratio, 17; 95\% confidence interval, 11-26) (2-5). In the CKiD cohort, the HR APOL1 genotype was associated with the glomerular phenotype in children younger than previously reported; previous studies have established that APOL1-associated FSGS is characterized by a tendency to present between ages 15 and 39 years old (7) and in FSGS-CT with median age 17 years $(13,23)(8)$. The median age of FSGS onset among HR children was 12 years in CKiD, which was older than other LR children with FSGS, a finding previously reported alongside the NEPTUNE children with nephrotic syndrome (15). Additionally, this study found a higher prevalence of prematurity among those with HR APOL1 compared to AA and non-AA children with FSGS, similar to findings with NEPTUNE that employed a different reference group, suggesting that the prematurity effect extends to non-AA children. 
It has been well established that AA children with FSGS have later onset disease than non-AA children and progress more rapidly to $\operatorname{ESRD}(23,24)$. In addition, AA children with nephrotic syndrome have a seven times greater risk of having been diagnosed with FSGS than Hispanics (Mexicans and other immigrants from Central America, but not Puerto Ricans and other Caribbean immigrants) (23), and this has been attributed to genetic factors. The present analysis suggest that among children with $\mathrm{CKD}$, AA children with HR APOL1 genotype present with later onset kidney disease, and have a higher prevalence of cardiovascular and metabolic comorbidities than patients with LR APOL1 genotype, despite a shorter duration of disease and the same diagnosis of FSGS. This would suggest that in children with HR genotypes, FSGS might have a different pathophysiology with the added influence of distinctive environmental factors (i.e., obesity and potentially birth history, toxin exposure, and/or additional genetic factors). This in turn may lead to a "vascular/ endothelial" phenotype clinically presenting as uncontrollable HTN and LVH leading to "enhanced" podocyte injury; in contrast, non-AA primary FSGS may be characterized by a "primary podocyte" phenotype, earlier onset, and slower CKD progression. It is unclear at present how G1 and G2 alleles may cause endothelial or podocyte damage, although the APOL1 protein forms pores in the lysosomal membrane, leading to pathogen lysis, and a variant APOL1 protein was shown in transgenic mice to induce direct tissue injury (25).

The role of APOL1 in the adult cardiovascular phenotype remains controversial. Ito et al. (6) using data from the Jackson Heart Study (JHS) and Women's Health Initiative (WHI) demonstrated that the APOL1 kidney risk alleles confer a twofold risk of cardiovascular events, without significant changes in left ventricular mass, whereas the Systolic Blood Pressure Intervention Trial (SPRINT) showed the absence of an APOL1 association with prevalent cardiovascular disease in a non-diabetic adult sample (26). It is possible that cardiovascular disease- $A P O L 1$ interaction may have an age-dependent relationship pattern, similar to one described in early- and late-onset forms of Alzheimer disease associated with apolipoprotein E, epsilon 4 allele, where subjects at both age spectrum (young-old) seem to be affected by the disease penetrance (27). It is unknown if similar age-dependent relationship pattern could also be seen in cardiovascular diseaseAPOL1 interaction.

Furth et al. (19) described cardiovascular disease risk factors in the CKiD cohort and found that only $18 \%$ of the cohort exhibited CRP values $>3 \mathrm{mg} / \mathrm{l}$ (independent of GFR). In our study, elevated CRP was not statistically different between HR and LR APOL1 children with FSGS, suggesting that inflammation is not a driving factor behind LVH. It is possible that differences in $\mathrm{LVH}$ are driven by a combination of $A P O L 1$ genotype, underlying metabolic status, obesity/BMI, HTN, and some other unrecognized socioeconomic or racial variables. Our results showed an excess of obesity among those with HR APOL1. Since obesity and metabolic dysregulation are risk factors for both cardiovascular disease and CKD onset and progression, managing weight prior to onset in order to prevent or delay disease may be an important clinical consideration. Future studies should seek to clarify whether the effect of $A P O L 1$ on CKD severity is related to obesity or whether these two outcomes are independently influenced by the HR profile. Our finding of increased cardiovascular and metabolic risks associated with HR genotype in children with $\mathrm{CKD}$ underscores the importance of early detection and need for more aggressive treatment of obesity, such as early therapy or adherence support, in this population.

It is also possible that the increased cardiovascular risk in the HR children is a consequence of the underlying glomerular disease (FSGS) severity and rate of progression. HTN-misattributed kidney disease in AA has been described in the adult literature (28). Given the cross-sectional nature of this study, future research efforts might investigate a large group of AA children with non-glomerular/congenital kidney disease to investigate the effect of HR APOL1 on cardiovascular risk, in the absence of podocyte injury and glomerular damage. Since the vast majority of HR children in CKiD had a glomerular disease (85\%), we were unable to assess the effect of APOL1 HR on cardiovascular risk among those with a non-glomerular condition.

This study has several limitations. First, the LR APOL1 subgroup with FSGS was predominantly non-AA. There were only 7 AA with LR APOL1 compared to 25 AA children with HR APOL1 and FSGS, thus highlighting the strong association between HR APOL1 and FSGS among AA children. Since other forms of glomerular disease among the AA LR APOL1 children, such as hemolytic uremic syndrome, familial nephritis, or SLE, were less common than FSGS in AA HR group, we chose to restrict our analysis to only those with FSGS to determine the effect of HR APOL1 among those with the same CKD pathology, which necessitated inclusion of non-AA children with FSGS. Although a minority of non-AA subjects were genotyped; all had LR APOL1 status, providing a justification for classifying non-AA children as LR. It is possible that some children were misclassified, particularly those of Hispanic ethnicity, although this misclassification would lead to more conservative estimates. Ideally, our study would have included a large group of LR and HR APOL1 AA children with FSGS. As such, we cannot completely discount genetic (non-APOL1) or metabolic factors related to race explaining these effects. A related limitation is the potential confounding effect of race and SES, given the disparate distributions of race between the exposed and unexposed groups. While several key SES variables were not associated with our exposure, 7-day antihypertensive adherence was related to HR APOL1. Inverse probability or exposure weights were used to account for potential differences in SES variables in adjusted analyses, but there may be other unmeasured SES variables possibly confounding these relationships. The third limitation was the lack of DNA for analysis in 59 subjects (28 subjects with glomerular disease), reducing statistical power. We did not characterize ancestry-informative markers, and therefore, we did not determine the fraction of African ancestry among subjects, although this information would likely not change any inferences, since the prevalence of HR APOL1 genotype in this study was nearly identical to that previously reported $(5,7,8)$. Finally, this study was limited in sample size and may be considered preliminary data for future research to build upon. These findings need to be replicated in larger populations in both children and adults. Despite these limitations, the association of HR APOL1 genotype 
with development of glomerular disease, later onset of FSGS and indications of increased cardiovascular risk among children were compelling.

In conclusion, this study suggests that $A P O L 1$-associated risks are not restricted to adults only and are present in young children as well. Indeed, in $\mathrm{CKiD}$, cardiovascular abnormalities were more common among AA children with HR APOL1. Targeting these established modifiable comorbidities, especially BMI, obesity, and HTN, may be a particularly important component in CKD management to delay ESRD in this HR population.

\section{AUTHOR CONTRIBUTIONS}

RW developed the idea for this research, participated in analysis and interpretation of the data, and wrote the manuscript. DN participated in the statistical design, analysis and interpretation of the data, and co-wrote the manuscript. SL, CW, JK participated in sample genotyping, analysis, and interpretation of the data, and edited the manuscript. KR and MS participated in analysis and interpretation of the data, and edited the manuscript. BW, SF, MM, CW, and FK participated in the design of the Chronic Kidney Disease in Children prospective cohort study (source of data in this manuscript) and analysis and interpretation of the data, and edited the manuscript.

\section{ACKNOWLEDGMENTS}

Data in this manuscript were collected by the Chronic Kidney Disease in Children prospective cohort study (CKiD) with clinical

\section{REFERENCES}

1. Bibbins-Domingo K, Pletcher MJ, Lin F, VittinghoffE, Gardin JM, Arynchyn A, et al. Racial differences in incident heart failure among young adults. $N$ Engl J Med (2009) 360:1179-90. doi:10.1056/NEJMoa0807265

2. Genovese G, Friedman DJ, Ross MD, Lecordier L, Uzureau P, Freedman BI, et al. Association of trypanolytic APOL1 variants with kidney disease in African Americans. Science (2010) 329(5993):841-5. doi:10.1126/ science.1193032

3. Freedman BI, Kopp JB, Langefeld CD, Genovese G, Friedman DJ, Nelson GW, et al. The apolipoprotein L1 (APOL1) gene and nondiabetic nephropathy in African Americans. JAm Soc Nephrol (2010) 21:1422-6. doi:10.1681/ ASN.2010070730

4. Tzur S, Rosset S, Shemer R, Yudkovsky G, Selig S, Tarekegn A, et al. Missense mutations in the APOL1 gene are highly associated with end stage kidney disease risk previously attributed to the MYH9 gene. Hum Genet (2010) 128(3):345-50. doi:10.1007/s00439-010-0861-0

5. Kopp JB, Nelson GW, Sampath K, Johnson RC, Genovese G, An P, et al. APOL1 genetic variants in focal segmental glomerulosclerosis and HIVassociated nephropathy. J Am Soc Nephrol (2011) 22:2129-37. doi:10.1681/ ASN.2011040388

6. Ito K, Bick AG, Flannick J, Friedman DJ, Genovese G, Parfenov MG, et al. Increased burden of cardiovascular disease in carriers of APOL1 genetic variants. Circ Res (2014) 114:845-50. doi:10.1161/CIRCRESAHA.114.302347

7. Parsa A, Kao WH, Xie D, Astor BC, Li M, Hsu CY, et al. APOL1 risk variants, race, and progression of chronic kidney disease. N Engl J Med (2013) 369(23):2183-96. doi:10.1056/NEJMoa1310345

8. Kopp JB, Winkler CA, Zhao X, Radeva MK, Gassman JJ, D’Agati VD, et al. Clinical features and histology of apolipoprotein L1-associated nephropathy in the FSGS clinical trial. J Am Soc Nephrol (2015) 26:1443-8. doi:10.1681/ ASN.2013111242 coordinating centers (Principal Investigators) at Children's Mercy Hospital and the University of Missouri - Kansas City (Bradley Warady, MD) and Children's Hospital of Philadelphia (Susan Furth, MD, Ph.D.), Central Biochemistry Laboratory (George Schwartz, MD) at the University of Rochester Medical Center, and data coordinating center at the Johns Hopkins Bloomberg School of Public Health (Alvaro Muñoz, Ph.D.). The CKiD Study is supported by grants from the National Institute of Diabetes and Digestive and Kidney Diseases, with additional funding from the Eunice Kennedy Shriver National Institute of Child Health and Human Development, and the National Heart, Lung, and Blood Institute (U01-DK-66143, U01-DK-66174, U01-DK-082194, and U01-DK-66116). This work was also supported by the National Cancer Institute (NCI) Intramural Research Programs. This project has been funded in part with federal funds from the National Cancer Institute, National Institutes of Health, under contract HHSN26120080001E. The content of this publication does not necessarily reflect the views or policies of the Department of Health and Human Services nor does mention of trade names, commercial products, or organizations imply endorsement by the U.S. Government. These data were presented in abstract form at the Annual Meeting of the American Society of Nephrology in 2013 and 2014.

\section{SUPPLEMENTARY MATERIAL}

The Supplementary Material for this article can be found online at http://journal.frontiersin.org/article/10.3389/fped. 2016.00122/full\#supplementary-material.

9. Hadtstein C, Schaefer F. Hypertension in children with chronic kidney disease: pathophysiology and management. Pediatr Nephrol (2008) 23:363-71. doi:10.1007/s00467-007-0643-7

10. Mitsnefes M, Ho PL, McEnery PT. Hypertension and progression of chronic renal insufficiency in children: a report of the North American Pediatric Renal Transplant Cooperative Study (NAPRTCS). J Am Soc Nephrol (2003) 14:2618-22. doi:10.1097/01.ASN.0000089565.04535.4B

11. Flynn JT, Mitsnefes M, Pierce C, Cole SR, Parekh RS, Furth SL, et al. Blood pressure in children with chronic kidney disease: a report from the Chronic Kidney Disease in Children Study. Hypertension (2008) 52:631-7. doi:10.1161/ HYPERTENSIONAHA.108.110635

12. Mitsnefes MM, Kimball TR, Kartal J, Witt SA, Glascock BJ, Khoury PR, et al. Progression of left ventricular hypertrophy in children with early chronic kidney disease: 2-year follow-up study. J Pediatr (2006) 149:671-5. doi:10.1016/ j.jpeds.2006.08.017

13. Kizer JR, Arnett DK, Bella JN, Paranicas M, Rao DC, Province MA, et al. Differences in left ventricular structure between black and white hypertensive adults: the Hypertension Genetic Epidemiology Network study. Hypertension (2004) 43(6):1182-8. doi:10.1161/01.HYP.0000128738.94190.9f

14. Kupferman JC, Aronson Friedman L, Cox C, Flynn J, Furth S, Warady B, et al. $\mathrm{BP}$ control and left ventricular hypertrophy regression in children with CKD. J Am Soc Nephrol (2014) 25(1):167-74. doi:10.1681/ASN.2012121197

15. Ng DK, Robertson CC, Woroniecki RP, Limou S, Gillies CE, Reidy KJ, et al. APOL1-associated glomerular disease among African-American children a collaboration of the Chronic Kidney Disease in Children (CKiD) and Nephrotic Syndrome Study Network (NEPTUNE) cohorts. Nephrol Dial Transplant (2016). doi:10.1093/ndt/gfw061

16. Furth SL, Cole SR, Moxey-Mims M, Kaskel F, Mak R, Schwartz G, et al. Design and methods of the Chronic Kidney Disease in Children (CKiD) prospective cohort study. Clin J Am Soc Nephrol (2006) 1(5):1006-15. doi:10.2215/ CJN.01941205 
17. National High Blood Pressure Education Program Working Group on High Blood Pressure in Children and Adolescents. The fourth report on the diagnosis, evaluation, and treatment of high blood pressure in children and adolescents. Pediatrics (2004) 114(2 Suppl 4th Report):555-76. doi:10.1542/ peds.114.2.S2.555

18. Khoury PR, Mitsnefes M, Daniels SR, Kimball TR. Age-specific reference intervals for indexed left ventricular mass in children. J Am Soc Echocardiogr (2009) 22(6):709-14. doi:10.1016/j.echo.2009.03.003

19. Furth SL, Abraham AG, Jerry-Fluker J, Schwartz GJ, Benfield M, Kaskel F, et al. Metabolic abnormalities, cardiovascular disease risk factors, and GFR decline in children with chronic kidney disease. Clin J Am Soc Nephrol (2011) 6(9):2132-40. doi:10.2215/CJN.07100810

20. Hidalgo G, Ng DK, Moxey-Mims M, Minnick ML, Blydt-Hansen T, Warady BA, et al. Association of income level with kidney disease severity and progression among children and adolescents with CKD: a report from the Chronic Kidney Disease in Children (CKiD) Study. Am J Kidney Dis (2013) 62(6):1087-94. doi:10.1053/j.ajkd.2013.06.013

21. Robins JM, Hernán MA, Brumback B. Marginal structural models and causal inference in epidemiology. Epidemiology (2000) 11(5):550-60. doi:10.1097/00001648-200009000-00011

22. Cole SR, Hernán MA. Constructing inverse probability weights for marginal structural models. Am J Epidemiol (2008) 168(6):656-64. doi:10.1093/aje/ kwn164

23. Sorof JM, Hawkins EP, Brewer ED, Boydstun II, Kale AS, Powell DR. Age and ethnicity affect the risk and outcome of focal segmental glomerulosclerosis. Pediatr Nephrol (1998) 12(9):764-8. doi:10.1007/s004670050542

24. Bonilla-Felix M, Parra C, Dajani T, Ferris M, Swinford RD, Portman RJ, et al. Changing patterns in the histopathology of idiopathic nephrotic syndrome in children. Kidney Int (1999) 55(5):1885-90. doi:10.1046/j.1523-1755.1999. 00408.x
25. Thomson R, Genovese G, Canon C, Kovacsics D, Higgins MK, Carrington M, et al. Evolution of the primate trypanolytic factor APOL1. Proc Natl Acad Sci U S A (2014) 111(20):E2130-9. doi:10.1073/pnas.1400699111

26. Langefeld CD, Divers J, Pajewski NM, Hawfield AT, Reboussin DM, Bild DE, et al. Apolipoprotein L1 gene variants associate with prevalent kidney but not prevalent cardiovascular disease in the Systolic Blood Pressure Intervention Trial (SPRINT). Kidney Int (2015) 87(1):169-75. doi:10.1038/ki.2014.254

27. Chartier-Harlin MC, Parfitt M, Legrain S, Pérez-Tur J, Brousseau T, Evans A, et al. Apolipoprotein E, epsilon 4 allele as a major risk factor for sporadic early and late-onset forms of Alzheimer's disease: analysis of the 19q13.2 chromosomal region. Hum Mol Genet (1994) 3(4):569-74. doi:10.1093/hmg/3.4.569

28. Skorecki KL, Wasser WG. Hypertension-misattributed kidney disease in African Americans. Kidney Int (2013) 83:6-9. doi:10.1038/ki.2012.369

Disclaimer: The content of this publication does not necessarily reflect the views or policies of the Department of Health and Human Services, and mention of trade names, commercial products, or organizations does not imply endorsement by the U.S. Government.

Conflict of Interest Statement: The authors declare that the research was conducted in the absence of any commercial or financial relationships that could be construed as a potential conflict of interest.

Copyright (c) 2016 Woroniecki, Ng, Limou, Winkler, Reidy, Mitsnefes, Sampson, Wong, Warady, Furth, Kopp and Kaskel. This is an open-access article distributed under the terms of the Creative Commons Attribution License (CC BY). The use, distribution or reproduction in other forums is permitted, provided the original author(s) or licensor are credited and that the original publication in this journal is cited, in accordance with accepted academic practice. No use, distribution or reproduction is permitted which does not comply with these terms. 